Neurocase

(1)

\title{
Effects of emotional face cueing on line bisection in neglect: A single case study
}

\section{Marco Tamietto, Luca Latini Corazzini , Lorenzo Pia , Marina Zettin , Maurizio Gionco \& Giuliano Geminiani}

To cite this article: Marco Tamietto , Luca Latini Corazzini , Lorenzo Pia , Marina Zettin , Maurizio Gionco \& Giuliano Geminiani (2005) Effects of emotional face cueing on line bisection in neglect: A single case study, Neurocase, 11:6, 399-404, DOI: $10.1080 / 13554790500259717$

To link to this article: http://dx.doi.org/10.1080/13554790500259717

\section{Published online: 16 Feb 2007.}

\section{Submit your article to this journal $\sqsubset$}

Џ Article views: 104

Q View related articles

Citing articles: 17 View citing articles 


\title{
Effects of emotional face cueing on line bisection in neglect: A single case study
}

\author{
MARCO TAMIETTO ${ }^{1,2}$, LUCA LATINI CORAZZINI ${ }^{1,2}$, LORENZO PIA $^{1,2}$, MARINA ZETTIN $^{2,3}$, MAURIZIO GIONCO $^{4}$ \\ and GIULIANO GEMINIANI ${ }^{1,2}$ \\ ${ }^{1}$ Department of Psychology, University of Turin, Italy \\ ${ }^{2}$ Center for Cognitive Science, University of Turin, Italy \\ ${ }^{3}$ Puzzle Center for Neurocognitive Rehabilitation, Turin, Italy \\ ${ }^{4}$ Department of Neurology and Rehabilitation, Mauriziano Hospital Umberto I, Turin, Italy
}

One patient with left neglect (FM) and four right brain-damaged controls were tested on a line bisection task with pictures of neutral and emotional faces as unilateral cues. We thus manipulated the attentional salience of the cues (higher for emotional and lower for neutral faces) while keeping constant their physical dimensions. Our findings showed that left emotional faces were more effective than left neutral faces in reducing bisection errors only in FM. These data indicate that in the neglected hemispace cues bias attention rather than simply altering the perceptual point of balance of the line in the horizontal plane.

\section{Introduction}

Patients with unilateral spatial neglect tend to ignore (i.e., neither react to nor search for) stimuli in the contralesional hemispace. A typical clinical test for the diagnosis of neglect is the line bisection task where subjects are asked to mark the midpoint of a horizontal line (Schenkenberg et al., 1980). Generally, the rightward bisection bias shown by neglect patients tends to decrease with unilateral left cues and is enhanced with unilateral right cues (Riddoch and Humphreys, 1983). This effect is usually obtained by placing either numbers or letters at the left or right end of the line and asking subjects to pay attention to them (reading them out) prior to bisection. Despite its empirical consistency, the explanation for the cueing effect is still the subject of debate. Indeed, several authors have explained cueing effects in terms of attentional mechanisms, whereas other authors have challenged this view, proposing a perceptual account (Fischer, 2001). The former hypothesis posits that a cue draws attention to that side, thereby rendering the cued side more salient. As a result of this increased

Received 15 March 2005; accepted 17 July 2005

This work was supported by a grant from the Ordine Mauriziano di Torino to Marco Tamietto.

We are grateful to Anna Berti for valuable comments on an earlier version of this manuscript. Thanks also to Patrizia Gindri for help in testing patients. Special thanks to Tiziana Zilli for technical support in mapping brain lesions with MRIcro. Giuliano, Lorenzo, Luca, and Marco wish to dedicate this study to the beloved memory of Michele Girardi: Take care, 'Don Michele'.

Address correspondence to Marco Tamietto, Dipartimento di Psicologia, Università di Torino, Via PO 14, 10123, Torino, Italy. E-mail: tamietto@psych.unito.it salience, the length of the cued side is overestimated and the mark is placed toward that side (Milner et al., 1992; Smania et al., 1998; Pouget and Driver, 2000; Olk and Harvey, 2002). Conversely, the latter interpretation points out that, since cues are placed beyond the true endpoint of the line, they extend the line's horizontal extent by several millimeters. This misperception of the line's length, induced by the alteration in the perceptual point of balance of the stimulus in the horizontal plane, explains the cueing effect without the need of postulating any attentional shift (Mattingley et al., 1993; Fischer, 1994).

Disentangling attentional from perceptual interpretations has been particularly problematic with unilateral cueing, as it is impossible to separate the relative contributions of an attentional manipulation from those caused by the alteration of the perceptual point of balance when the same cue is used. One way to empirically contrast attentional and perceptual accounts in a line bisection task with unilateral cues is to vary the attentional salience of the cues while keeping their physical extent constant.

Previous studies showed that emotional faces have a special advantage over neutral faces in summoning spatial attention (Hansen and Hansen, 1988; Öhman et al., 2001; Vuilleumier et al., 2001; Vuilleumier \& Schwartz, 2001). This is probably due to the high biological and social value of emotional stimuli for survival and adaptive behavior (Adolphs, 2002). In the present study we have thus used neutral and emotional faces of the same size as unilateral left or right cues to address the issue of the underlying mechanisms, attentional or perceptual, responsible for the cueing effect in neglect. By considering the foregoing alternative explanations of the cueing effect, we can formulate the following hypotheses: if the cueing effect works through biasing 
attention, we should observe a modulation of the line bisection error as a function of the salience conveyed by the cues. That is, emotional cues should be more effective than neutral face cues and this should be true for contralesional cues in particular. If, conversely, the cueing effect is caused by a perceptual alteration of the point of balance, we should expect a comparable influence of all cues, whatever their specific content, as they all have the same dimensions.

\section{Method}

\section{Case report}

We studied patient FM, a 73-year-old, right-handed Caucasian woman with chronic unilateral right brain damage from neoplasia (Figure 1). In August 2002 FM was admitted to the Mauriziano Hospital Umberto I of Turin because of sudden and acute hemicranias in the right posterior hemisphere resistant to analgesic therapies. On neurological examination she had mild left arm paresis with decreased touch sensation. There was no gaze palsy but she tended to orient her eyes and head to the right. A CT scan performed the same day demonstrated a low attenuation area in the right parietal lobe, consistent with neoplasia. Subsequent T1 and T2 weighted MRI scans performed 5 days later confirmed the diagnosis and showed a prevailing involvement of the underlying white matter. When tested in the following days she showed severe left neglect in all the tasks composing a battery for the diagnosis of neglect, which included: the line cancellation test (Albert, 1973), the letter cancellation test (Diller \& Weinberg, 1977), a sentence-reading test, and the line bisection test (Schenkenberg et al., 1980). Face perception and emotional processing from facial expressions were also tested using, respectively, the Benton Facial Recognition Test (BFRT) (Benton et al., 1983) and the Ekman 60 Faces Test (ESFT) (Young et al., 2002). FM was aware of her deficits and cooperative.

\section{Control subjects}

Four patients with unilateral right brain damage and no sign of neglect in any of the tasks served as control group.

Demographic, clinical, and neuropsychological data of FM and the control patients are summarized in Table 1. All patients provided written informed consent approved by the Ethical Committee of the University of Turin, Italy.

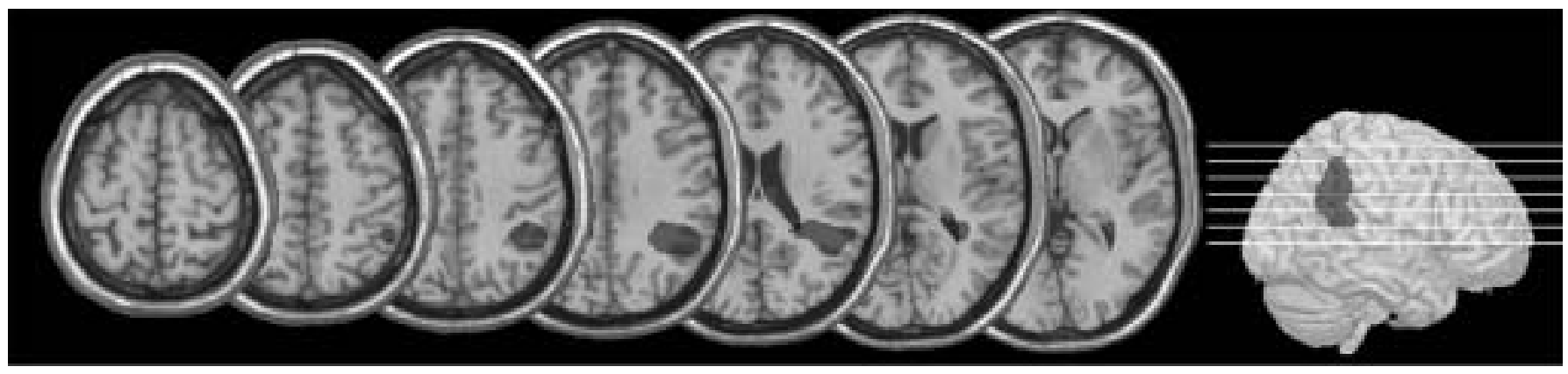

Fig. 1. Lesion reconstruction in the neglect patient FM, mapped using MRIcro software (Rorden \& Brett, 2000).

Table 1. Demographic, clinical, and neuropsychological data of the patients

\begin{tabular}{|c|c|c|c|c|c|c|c|c|c|c|c|c|c|}
\hline Patient & Sex & Age & Lesion & Etiology & Onset (days) & MMSE & VFD & SRT & $\begin{array}{l}\text { LBT M } \\
\quad(\mathrm{SD})\end{array}$ & Diller L-R & Albert L-R & BFRT* & ESFT \\
\hline FM & $\mathrm{F}$ & 73 & T-O-P & $\mathrm{N}$ & 10 & 27.07 & $2-3$ & $1 / 5$ & $45.6(14)$ & $0-23$ & $0-11$ & 41 & 45 \\
\hline FG & $\mathrm{F}$ & 77 & Fr-T & I & 22 & 26.03 & $0-0$ & $5 / 5$ & $0.7(0.93)$ & $48-50$ & $18-18$ & 44 & 51 \\
\hline $\mathrm{MD}$ & $\mathrm{M}$ & 71 & BG-EC-Ins & I & 13 & 25.7 & $0-0$ & $5 / 5$ & $3.15(1.93)$ & $52-52$ & $18-18$ & 47 & 50 \\
\hline MG & $\mathrm{M}$ & 60 & Fr-T-P & I & 213 & 30 & $0-0$ & $5 / 5$ & $3.1(2.4)$ & $47-49$ & $18-18$ & 43 & 46 \\
\hline $\mathrm{BN}$ & $\mathrm{M}$ & 62 & IC-Ins-BG & I & 21 & 30 & $0-0$ & $5 / 5$ & $2.5(3.1)$ & $52-51$ & $18-18$ & NA & NA \\
\hline
\end{tabular}

Abbreviations: BFRT = Benton Facial Recognition Test (cut-off $=37$ ), *the stimuli were arranged centrally along the vertical meridian; ESFT = Ekman 60 Faces Test (cut-off = 42); Etiology: I = Ischemia, N = Neoplasia; LBT = line bisection test (mean (standard deviation) rightward deviation in mm on 10 lines); Lesion: $\mathrm{BG}=$ basal ganglia; $\mathrm{EC}=$ external capsule; $\mathrm{Fr}=$ frontal; $\mathrm{IC}=$ internal capsule; Ins = insula; $\mathrm{O}=$ occipital; $\mathrm{P}=$ parietal; $\mathrm{T}=$ temporal; $\mathrm{MMSE}=\mathrm{Mini}$ Mental State Examination (Folstein, Folstein, \& McHugh, 1975); NA = not available; SRT = sentence reading test (5 sentences); VFD = visual field defects within the contralesional hemispace measured on the standard confrontation test (the two values refer to the upper and lower quadrants, respectively), scores ranged from normal vision (0) to severe defect (3) (Bisiach, Cappa \& Vallar, 1983); Diller = cancelled targets in the left hemispace (L) - cancelled targets in the right hemispace $(\mathrm{R})$, (number of total targets per hemispace $=52$ ); Albert $=$ cancelled targets in the left hemispace $(\mathrm{L})-$ cancelled targets in the right hemispace $(\mathrm{R})$, (number of total targets per hemispace $=18$ ). 


\section{Stimuli and procedure}

Black lines $(180 \times 1 \mathrm{~mm})$ were used as stimuli. Each line was placed horizontally and centrally on a separate sheet of paper. Black-and-white photographs of 8 different actors taken from Ekman's series (Ekman and Friesen, 1976) (4 males; $60 \times 40 \mathrm{~mm}$ ) with either a neutral, happy, or angry expression served as cues and could be present at the right or left end of the line, or absent. When present, the cues were of three different types: a picture of a neutral, happy or angry face. The neutral faces provided an appropriate and extremely conservative control condition because, like emotional faces, they belong to the same stimulus category, have the same personal identity (as the same actors presented with a neutral expression were also shown with an emotional expression), and share the same dimensions, elementary components and global configuration. Overall there were seven possible stimulus conditions: three different cue types on the left, three on the right, and the no cue condition where only the lines were present.

The midpoint of each line was aligned with the patient's mid-sagittal plane. The task was to mark the midpoint of the line using the right hand. Before doing so, patients were asked to pay attention to both sides of the line and to put a mark under the cues, if any, whatever was depicted. Thus emotional content in cues was completely irrelevant to the task and could be ignored. Each patient bisected 20 lines per condition for a total of 140 lines randomly presented and divided into 4 subsequent blocks of 35 lines each.

Errors in line bisection for each patient and condition were measured to the nearest millimeter. Errors to the right of the objective midpoint were given a positive and those to the left a negative value.

\section{Results}

Figure 2 reports the performances of patient FM (a) and of the control group (b) as a function of the seven cue conditions.

\section{Cueing Effect}

\section{Patient FM}

In order to evaluate whether the cueing manipulation was effective in FM, all three left cue conditions were collapsed into a single left cue condition, and vice-versa for the right cue conditions. An ANOVA was then performed on error scores with the within-subjects factor of Cue Position (no cue (NC), left cue (LC), and right cue (RC)).

There was a significant main effect of Cue Position $(\mathrm{F}(2,38)=146.54, p<.001)$. Post-hoc tests (Newman-Keuls for all comparisons henceforth) revealed that the rightward bias shown with no cue decreased in the LC condition and increased in the RC condition $(p<.0002$ and $p<.007$, respectively).

\section{Control group}

We performed an ANOVA on mean error scores for the control group with the same factors and levels considered for FM. The main effect of Cue Position was statistically significant $(\mathrm{F}(2,6)=24.36, p<.001)$. Post hoc tests revealed that bisections were placed significantly to the left in the left cue condition compared with no cue and right cue conditions $(p<$ .014 and $p<.002$, respectively). Similarly, right cues resulted in increased rightward errors as compared to the no cue and left cue conditions $(p<.013)$.
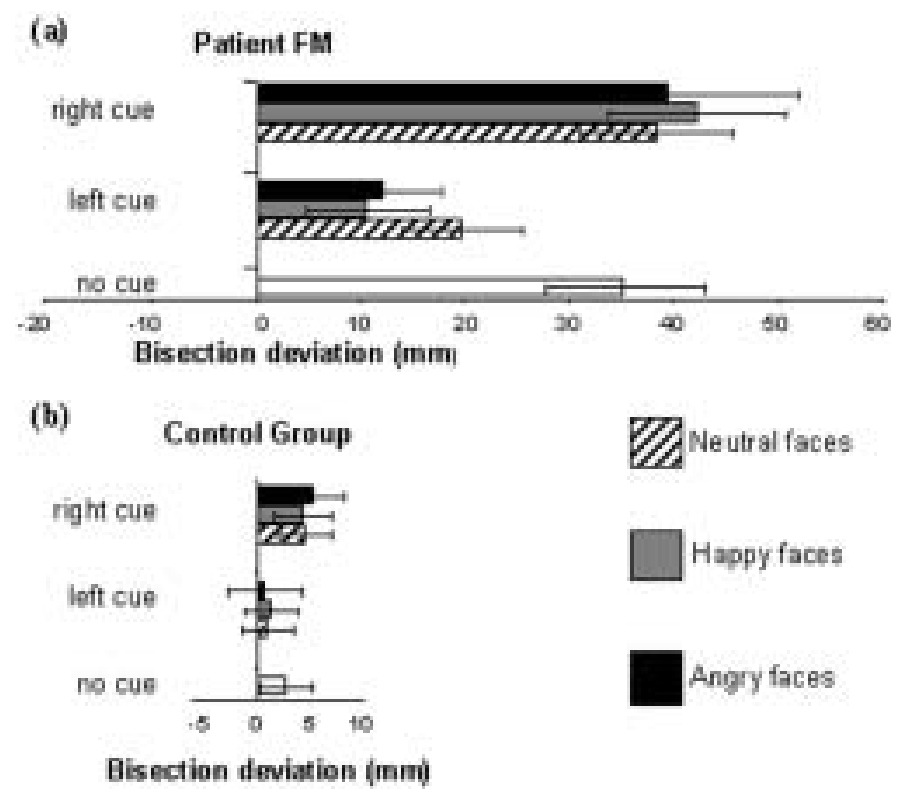

Fig. 2. Mean error (mm) in the line bisection task for FM (a) and the Control Group (b) by cue position and cue type. Rightward errors are coded as positive, leftward as negative. Error bars indicate standard deviation. 
These results show that the cueing manipulation was effective in modulating bisection performance in patient FM, as well as in the control group. Obviously, the absolute rightward error and effect size was much greater in the neglect patient than in the control group.

\section{Emotional effect}

\section{Patient FM}

A $2 \times 3$ ANOVA was computed with the within-subjects factors of Cue Position (left vs. right) and Cue Type (neutral (NF), happy (HF), and angry face (AF)). The emotional effect was thus tackled by comparing the performance in the $\mathrm{NF}$ condition with that in the emotional face conditions (i.e., $\mathrm{HF}$ and $\mathrm{AF}$ ). The effect of Cue Position was significant $(\mathrm{F}(1,19)=412.7, p<.001)$, further indicating an efficient cueing manipulation. The effect of Cue Type was not significant but the Cue Position X Cue Type interaction clearly was $(\mathrm{F}(2,38)=6.77, p<.003)$. Post-hoc tests on the interaction showed that bisections with left HF and AF cues were placed significantly further to the left by reference to the left NF condition ( $p<.005$ for both comparisons). The left HF and AF conditions did not differ from each other $(p>.5)$. There was no significant difference among right cues $(p>.25$ for all comparisons).

\section{Control group}

The ANOVA on mean errors showed only a significant effect of Cue Position $(\mathrm{F}(1,3)=57.89, p<.004)$, indicating a comparable influence of all left cues in shifting bisections leftward and of all right cues in increasing rightward bias. Importantly, no specific effect due to the emotional value of the cues was found.

\section{Discussion}

Prior studies testing neglect patients with the line bisection task documented an influence of left cues in reducing the rightward bias, and an enhancement of the rightward bisection error with unilateral right cues (Riddoch and Humphreys, 1983; Nichelli et al., 1989). The mechanisms responsible for this cueing effect are, however, still unclear. The two major theoretical accounts have proposed both an attentional and a perceptual explanation (Fischer, 2001). Attentional explanations assume that cues call for attention, thereby increasing the salience of the cued side. This modulation of spatial attention 'pulls' the patients' bisection behavior to the cued side (Milner et al., 1992; Olk and Harvey, 2002). Perceptual interpretations, on the other hand, consider that cueing effects might best be explained by the alteration of the perceptual point of balance of the stimulus toward the cued side (Mattingley et al., 1993; Fischer, 1994).

In the present study, we have addressed the issue of the mechanisms underlying cueing effects by varying the attentional salience of the cues while keeping their physical dimensions constant. This cueing paradigm has not earlier been used with neglect patients. Nor has a comparison between right braindamaged patients with and without neglect been performed before. In keeping with previous studies, control patients without neglect showed a weak bisection bias to the right in the no cue condition, whereas the neglect patient FM revealed a larger rightward bias in the same condition (Machado and Rafal, 1999; Olk and Harvey, 2002). The cueing manipulation per se was also evident in both patient FM and the control group, with left cues reducing, and right cues increasing the bisection bias.

More interestingly, the various cueing conditions affected the subjectively perceived length of the lines in different ways depending on the type of patients and on the side where cues appeared. In control subjects with right hemisphere lesions, the specific content of the cues did not affect line bisection either with left of right cues. Indeed, the Cue Type factor and the Cue Position X Cue Type interaction were both nonsignificant. In patient FM, by contrast, left happy and angry faces were more effective than left neutral faces in reducing the rightward directional bias. Importantly, the modulation of emotional faces on line bisection was confined to the left (neglected) hemispace and occurred even though the content of the cues was irrelevant to the task. This is unlikely to be the result of some low-level perceptual differences among stimuli. Indeed, all stimuli shared the same dimensions, and had the same elementary components and global configuration. Our findings are also unlikely to be the result of differences in the personal identity of the faces, as the same actors presented with a neutral expression were also shown with an emotional expression. Furthermore, FM appeared to be hemianopic in the standard confrontation test (see Table 1). Even though the assessment of primary visual field deficits in neglect patients through clinical tests is not conclusive, it could be argued that FM at least behaved as if she had hemianopia. This suggests that at the very moment of bisection (i.e., when the patient was certainly foveating the tip of the pen), left cues were prevented from vision, thus rendering unlikely any influence related to possible low-level perceptual factors.

The different influence of the left cues in a neglect patient as a function of their emotional and attentional value, clearly supports the idea that the cueing effect works through biasing attention rather than by altering the perceptual point of balance (at least in the contralesional hemispace). This view is also in line with other studies that used invisible (Harvey et al., 2000; Olk and Harvey, 2002) or symmetrical cues (Jeerakathil and Kirk, 1994; Kashmere and Kirk, 1997). Conversely, an interpretation based on the alteration of the perceptual point of balance would predict a comparable influence of all unilateral left cues in reducing the rightward bias, whatever their specific content, which was not the case. Arguably, in the left (contralesional) hemispace of neglect patients, where the systems for normal length perception and cueing effects may have been damaged by right parietal 
lesions, the attentional manipulation becomes apparent. Conversely, in the right (ipsilesional) hemispace of neglect patients, and in patients without neglect where the attentional system is relatively preserved, the attentional call due to the salience of emotional faces may be overridden by perceptual factors. An alternative, and less likely, interpretation might be that the enhanced influence of left as compared to right emotional cues indicates that cueing effects work differently, depending on whether they are concomitant with lateralized attentional deficits. An attentional mechanism for contralesional cues in neglect patients could thus be hypothesized, whereas a perceptual mechanism could account for the influence of ipsilesional cues in neglect patients and for both left and right cues in subjects with right hemisphere lesions without neglect. This latter interpretation, however, is much less parsimonious than the former and needs further empirical support to be taken into consideration.

Prior behavioral findings have suggested that the distribution of spatial attention in patients with visual extinction and spatial neglect may be influenced by emotional faces (Vuilleumier and Schwartz, 2001). Here we show for the first time in a line bisection task that this influence on spatial attention has additional behavioral consequences in promoting salience and access to action in the contralesional hemispace. The privileged processing of emotional faces, even when task-irrelevant, suggests that (a) the emotional content of the faces can be encoded in the neglected hemispace, (b) the results of such encoding can shift the attentional focus, and (c) this modulation of spatial attention guides subsequent orienting behaviors and motor outputs to salient left-sided events.

However, within an attentional account of the emotional cueing effect, the specific mechanisms through which facial expressions increased the salience of the cued left side of the line still remain unclear. Indeed, it has been shown that the attentional call of emotional faces may directly modulate early visual processing and peripheral stimulus detection across the visual field (Morris et al., 1998; Vuilleumier and Schwartz, 2001; Vuilleumier et al., 2004). Alternatively, it is also possible that emotional cues exerted an influence on the maintenance in the spatial working memory (SWM) of previously inspected locations, therefore rendering more salient and/or efficient the remembering of the left side of the line. This would be in keeping with recent studies showing that non-lateralized SWM deficits are often associated with, and can exacerbate, spatial neglect (Husain et al., 2001; Wojciulik et al., 2001; Driver and Husain, 2002; Malhotra et al., 2004, 2005).

The involvement of specific limbic structures (amygdala, cingulate, and orbitofrontal cortex) in processing emotional information has been repeatedly demonstrated with different techniques and in various neurological conditions (Adolphs, 2002). Interestingly, even in neglect patients, unattended and unseen affective facial expressions underwent substantial neural processing and activated the amygdala (Vuilleumier et al., 2002). Thus such limbic activation is the most probable candidate for the attentional enhancement due to emotional stimuli observed here and, more specifically, suggests the role of the amygdala in regulating cortical processing. Indeed, the amygdala might be activated by information from the contralesional hemispace through direct pathways from the thalamus bypassing the primary visual cortex or via ventral occipito-temporal pathway (de Gelder et al., 1999; Morris et al., 1999; Morris et al., 2001). Then, the amygdala might exert its modulatory role by direct projections to visual areas (Vuilleumier et al., 2004), via reciprocal connections to the anterior attentional network in the dorsolateral prefrontal cortex (Pessoa et al., 2002), or through complex interactions with other medial temporal structures (e.g., the hippocampus) and prefrontal areas involved in memory functions (Peinadomanzano, 1990; McGaugh, 2000; Roozendaal et al., 2004). This triple involvement of limbic structures in emotional processing, spatial attention, and memory might have given more weight to emotional faces competing for attention and action. In this context, it is worth noting that the foregoing limbic areas were bilaterally intact in patient FM, who consistently performed in the normal range in the ESFT.

Overall, our findings support the neuro-functional model of spatial attention put forth by Mesulam $(1999 ; 2002)$ where the salience of extrapersonal events encoded by limbic areas is regarded as particularly influential on attentional shift. In the model, spatial attention depends on a neural network including mainly the posterior parietal, dorsolateral frontal, and cingulated cortex. Although all these components are collectively engaged in specifying whether an external event will attract attention, the parietal cortex is more involved in providing a sensory representation of the extrapersonal space, the dorsolateral frontal cortex subserves exploratory motor plans, and the cingulated and limbic components play a critical role in identifying the motivational relevance of extrapersonal events. Even though the role of limbic areas is the least well understood, our results suggest that intact limbic and frontal structures might still mediate attentional shift and motor planning in spite of parietal damage and spatial neglect.

\section{References}

Adolphs R. Recognizing emotion from facial expressions: Psychological and neurological mechanisms. Behavioral and Cognitive Neuroscience Reviews 2002; 1: 21-61.

Albert ML. A simple test for visual neglect. Neurology 1973; 23: 658-64.

Benton AL, Hamsher KS, Varney NR, Spreen O. Contributions to neuropsychological assessment: A clinical manual. Oxford University Press: New York, USA, 1983.

Bisiach E, Cappa S, Vallar G. Guida all'esame neuropsicologico. Milano. Italy: Raffaello Cortina Editore, 1983.

de Gelder B, Vroomen J, Pourtois G, Weiskrantz L. Non-conscious recognition of affect in the absence of striate cortex. NeuroReport 1999; 10: 3759-63.

Diller L, Weinberg J. Hemi-inattention in rehabilitation: The evolution of a rational remediation program. In: EA, Weinstein, RP, Friedland, editors. Hemi-inattention and hemisphere specialization. New York, USA: Raven Press, 1977; 63-82. 
Driver J, Husain M. The role of spatial working memory deficits in pathological search by neglect patients. In: HO, Karnath, D, Milner, G, Vallar, editors. The cognitive and neural bases of spatial neglect. Oxford, UK: Oxford University Press, 2002; 351-64.

Ekman P, Friesen W. Pictures of facial affect. Palo Alto, CA: Consulting Psychologists Press, 1976.

Fischer MH. Less attention and more perception in cued line bisection. Brain and Cognition 1994; 25: 24-33.

Fischer MH. Cognition in the bisection task. Trends in Cognitive Science 2001; 5: 460-62.

Folstein NL, Folstein SE, McHugh PR. Mini mental state examination: A practical method for grading the cognitive state of patients for the clinician. Journal of Psychiatric Research 1975; 12: 189-98.

Hansen CH, Hansen RD. Finding the face in the crowd: An anger superiority effect. Journal of Personality and Social Psychology 1988; 54: 917-24.

Harvey M, Pool TD, Robertson MJ, Olk B. Effects of visible and invisible cueing procedures on perceptual judgements in young and elderly subjects. Neuropsychologia 2000; 38: 22-31.

Husain M, Mannan S, Hodgson T, Wojciulik E, Driver J, Kennard C. Impaired spatial working memory across saccades contributes to abnormal search in parietal neglect. Brain 2001; 124: 941-52.

Jeerakathil TJ, Kirk MA. A representation vertical bias. Neurology 1994; 44: 703-06

Kashmere JL, Kirk MA. The complex interaction of normal biases in line bisection. Neurology 1997; 49: 887-89.

Machado L, Rafal RD. Ipsilesional line bisection bias in patients with chronic parietal lesions. NeuroReport 1999; 10: 3143-48.

Malhotra P, Jäger HR, Parton A, Greenwood R, Playford ED, Brown MM, Driver J, Husain M. Spatial working memory capacity in unilateral neglect. Brain 2005; 128: 424-35.

Malhotra P, Mannan S, Driver J, Husain M. Impaired spatial working memory: One component of the visual neglect syndrome?. Cortex 2004; 40: 667-76.

Mattingley JB, Pierson JM, Bradshaw JL, Phillips JG, Bradshaw JA. To see or not to see: The effects of visible and invisible cues on line bisection judgements in unilateral neglect. Neuropsychologia 1993; 31 : 1201-15.

McGaugh JL. Memory-a century of consolidation. Science 2000; 287: $248-51$

Mesulam M. Spatial attention and neglect: Parietal, frontal and cingulated contributions to the mental representation and attentional targeting of salient extrapersonal events. Philosophical Transactions of the Royal Society of London Series B-Biological Sciences 1999; 354: 1325-46.

Mesulam M. Functional anatomy of attention and neglect: From neurons to networks. In: HO, Karnath, D, Milner, G, Vallar, editors. The cognitive and neural bases of spatial neglect. Oxford, UK: Oxford University Press, 2002; 33-46.

Milner AD, Brechmann M, Pagliarini L. To halve and to halve not: An analysis of line bisection judgements in normal subjects. Neuropsychologia 1992; 30: 515-26.

Morris JS, de Gelder B, Weiskrantz L, Dolan RJ. Differential extrageniculostriate and amygdala responses to presentation of emotional faces in a cortically blind field. Brain 2001; 124: 1241-52.
Morris JS, Friston KJ, Buchel C, Frith CD, Young AW, Calder AJ, Dolan RJ. A neuromodulatory role for the human amygdala in processing emotional facial expressions. Brain 1998; 121: 47-57.

Morris JS, Öhman A, Dolan RJ. A subcortical pathway to the right amygdala mediating "unseen" fear. Proceedings of the National Academy of Sciences of the United States of America 1999; 96: 1680-85.

Nichelli P, Rinaldi M, Cubelli R. Selective spatial attention and length representation in normal subjects and in patients with unilateral spatial neglect. Brain and Cognition 1989; 9: 57-70.

Öhman A, Lundqvist D, Esteves F. The face in the crowd revisited: A threat advantage with schematic stimuli. Journal of Personality and Social Psychology 2001; 80: 381-96.

Olk B, Harvey M. Effects of visible and invisible cueing on line bisection and Landmark performance in hemispatial neglect. Neuropsychologia 2002; 40: 282-90.

Peinadomanzano MA. The role of the amygdala and the hippocampus in working memory for spatial and nonspatial information. Behavioural Brain Research 1990; 38: 117-34.

Pessoa L, Kastner S, Ungerleider LG. Attentional control of the processing of neutral and emotional stimuli. Cognitive Brain Research 2002; 15: $31-45$.

Pouget A, Driver J. Relating unilateral neglect to the neural coding of space. Current Opinion in Neurobiology 2000; 10: 242-249.

Riddoch MJ, Humphreys GW. The effect of cueing on unilateral neglect. Neuropsychologia 1983; 2: 589-99.

Roozendaal B, McReynolds JR, McGaugh JL. The basolateral amygdala interacts with the medial prefrontal cortex in regulating glucocorticoid effects on working memory impairment. Journal of Neuroscience 2004; 24: 1385-92.

Rorden C, Brett M. Stereotaxic display of brain lesions. Behavioural Neurology 2000; 12: 191-200.

Schenkenberg T, Bradford DC, Ajax ET. Line bisection and unilateral visual neglect in patients with neurological impairments. Neurology 1980; 30: 509-17.

Smania N, Martini M, Gambina G, Tomellari G, Palamara A, Natale E, Marzi C. The spatial distribution of visual attenton in hemineglect and extinction patients. Brain 1998,; 21: 1759-70.

Vuilleumier P, Armony JL, Clarke K, Husain M, Driver J, Dolan RJ. Neural response to emotional faces with and without awareness: Event-related fMRI in a parietal patient with visual extinction and spatial neglect. Neuropsychologia 2002,; 40: 2156-66.

Vuilleumier P, Armony JL, Driver J, Dolan RJ. Effects of attention and emotion on face processing in the human brain: an event-related fMRI study. Neuron 2001; 30: 829-41.

Vuilleumier P, Richardson MP, Armony JL, Driver J, Dolan RJ. Distant influences of amygdala lesion on visual cortical activation during emotional face processing. Nature Neuroscience 2004; 7: 1271-78.

Vuilleumier P, Schwartz S. Emotional facial expressions capture attention. Neurology 2001; 56: 153-58.

Wojciulik E, Husain M, Clarke K, Driver J. Spatial working memory deficit in unilateral neglect. Neuropsychologia 2001; 39: 390-96.

Young, AW, Perrett, D, Calder, A, Sprengelmeyer, R; Ekman, P. Facial Expression of Emotion-Stimuli and Tests (FEEST). Bury St. Edmunds: Thames Valley Test Company., 2002. 